\title{
The compression type of coronary artery motion in patients with ST-segment elevation acute myocardial infarction and normal controls: a case-control study
}

\author{
Aiden JC O'Loughlin ${ }^{1 *}$, Karen Byth ${ }^{2}$, John K French ${ }^{4}$, David AB Richards ${ }^{4}$, Annemarie Hennessy ${ }^{1}$, \\ A Robert Denniss ${ }^{1,3,5}$, Pramesh Kovoor ${ }^{3}$
}

\begin{abstract}
Background: Prediction of the location of culprit lesions responsible for ST-segment elevation myocardial infarctions may allow for prevention of these events. A retrospective analysis of coronary artery motion (CAM) was performed on coronary angiograms of 20 patients who subsequently had ST-segment elevation myocardial infarction treated by primary or rescue angioplasty and an equal number of age and sex matched controls with normal angiograms.
\end{abstract}

Findings: There was no statistically significant difference between the frequency of CAM types of the ST-segment elevation acute myocardial infarction and control patients $(p=0.97)$. The compression type of CAM is more frequent in the proximal and mid segments of all three coronary arteries. No statistically significant difference was found when the frequency of the compression type of CAM was compared between the ST-segment elevation acute myocardial infarction and control patients for the individual coronary artery segments $(p=0.59)$.

Conclusion: The proportion of the compression type of coronary artery motion for individual artery segments is not different between patients who have subsequent ST-segment elevation myocardial infarctions and normal controls.

\section{Introduction}

The three-dimensional motion of the heart is characterized by rotation (around the centre of gravity), radial displacement (towards or away from the center of gravity), and translational motion (displacement parallel to its long axis) [1]. The total translational motion of the left ventricle is on average $2.2 \mathrm{~cm}$ and is such that motion occurs most at the base and least at the apex of the heart [2].

Motion of individual segments of coronary arteries reflects the motion of the underlying myocardium. The classification system for different patterns of coronary artery motion (CAM) used in this study is derived from a system where CAM was classified into 10 patterns,

* Correspondence: A.OLoughlin@uws.edu.au

'University of Western Sydney, Sydney, Australia

Full list of author information is available at the end of the article which were grouped into 3 types: (1) compression type: the length of the arterial segment is shortened without vertical deviation of the artery; (2) displacement type: the location of the coronary artery shifts without change of the length or shape of the arterial segment; and (3) bend type: the coronary artery flexes into a curve [3].

The compression type of CAM for individual artery segments is associated with stenosis [3] and is a predictor of segments containing the culprit lesion responsible for ST-segment elevation myocardial infarctions (STEMIs) [4]. The compression type of CAM has recently been shown to be strongly associated with segments containing the culprit lesion in STEMI patients after successful fibrinolysis [5].

The hypothesis to be tested in this study is that the compression type of CAM is more likely to be present in patients who have subsequent STEMI than in age
Ciomed Central

(ㄷ) 2011 O'Loughlin et al; licensee BioMed Central Ltd. This is an open access article distributed under the terms of the Creative Commons Attribution License (http://creativecommons.org/licenses/by/2.0), which permits unrestricted use, distribution, and reproduction in any medium, provided the original work is properly cited. 
and sex matched control patients with normal coronary angiograms.

\section{Methods}

Twenty patients were identified who had coronary angiography after March 1998 and subsequently represented with a STEMI. STEMI was defined as ischemic chest pain with ST segment elevation of $1 \mathrm{~mm}$ in 2 contiguous limb leads or $2 \mathrm{~mm}$ in 2 contiguous chest leads. Patients were excluded if they had previous coronary artery bypass surgery or had stent thrombosis as the cause of STEMI. Twenty age and sex matched control patients were identified with normal coronary angiograms.

The CAM patterns of coronary segments were assessed retrospectively in both the STEMI and control patients. For the STEMI patients, the coronary angiography performed before the STEMI was used. The assessment was made blinded to the location of the future culprit segment. The CAM classification of Konta and Bett [3] was used. A schematic of this classification is shown in Figure 1. All three coronary arteries were assessed using all available views. In a single view, a visual comparison was made between the coronary segment at the start and end of systole. A single pattern of motion was assigned in each view. Each segment was then assigned a CAM pattern by synthesizing the assignment for all available views. The patterns of CAM were grouped into the compression type and noncompression type (the bend and displacement types).

Assessment of CAM was made in up to fourteen segments of the coronary arterial tree. The segments were given a numerical label as shown in Figure 2.

Clinical risk factors (hypertension, diabetes, smoking, family history and hypercholesterolemia) of all patients were obtained from the medical records.

Chi-squared tests were used for comparison of frequencies between groups. All statistical analyses were performed using Stata (version 10.0, StataCorp, College Station, TX).

The Royal Prince Alfred Hospital Ethics Review Committee approved the research protocol (reference X100159). The research protocol did not include obtaining patient consent.

\section{Results}

The demographics for the STEMI and control patients are shown in Table 1. The frequency of each pattern and type of CAM for all the segments of both the STEMI and control patients are shown in Table 2. 67\% of segments in both the STEMI and control patients had a non-compression type of CAM. The bend type was present in $44 \%$ of segments in the STEMI patients and in $47 \%$ of segments in the control patients.

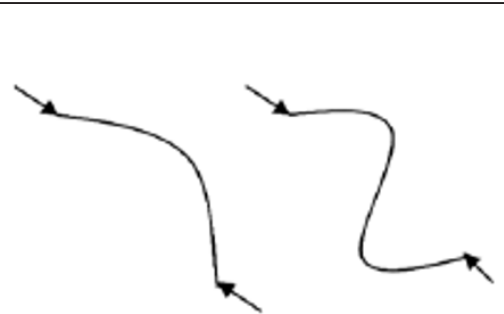

Bend
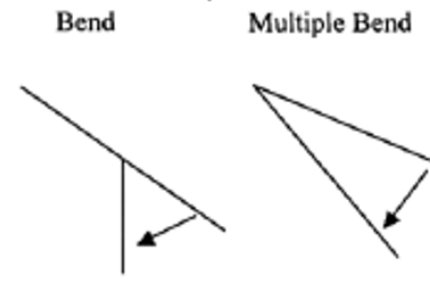

Hinge
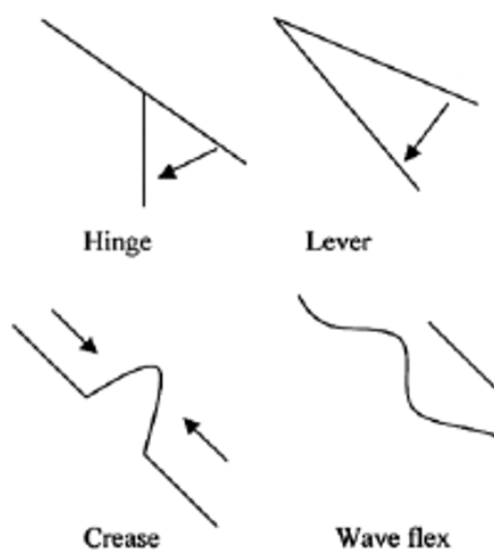

Wave flex

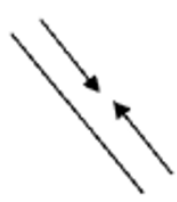

Compression

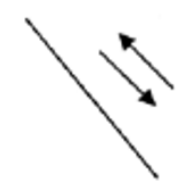

Lineal D

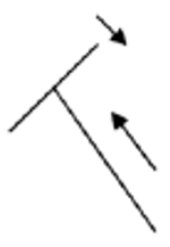

Ostial C

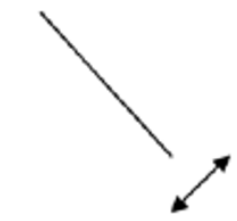

Parallel D

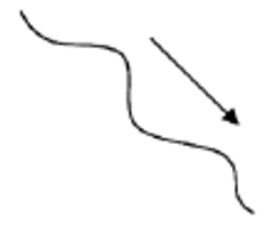

Figure 1 Classification of coronary artery motion. Lines illustrate the coronary artery segment and arrows show the direction of coronary artery motion. $\mathrm{C}=$ compression, $\mathrm{D}=$ displacement. 


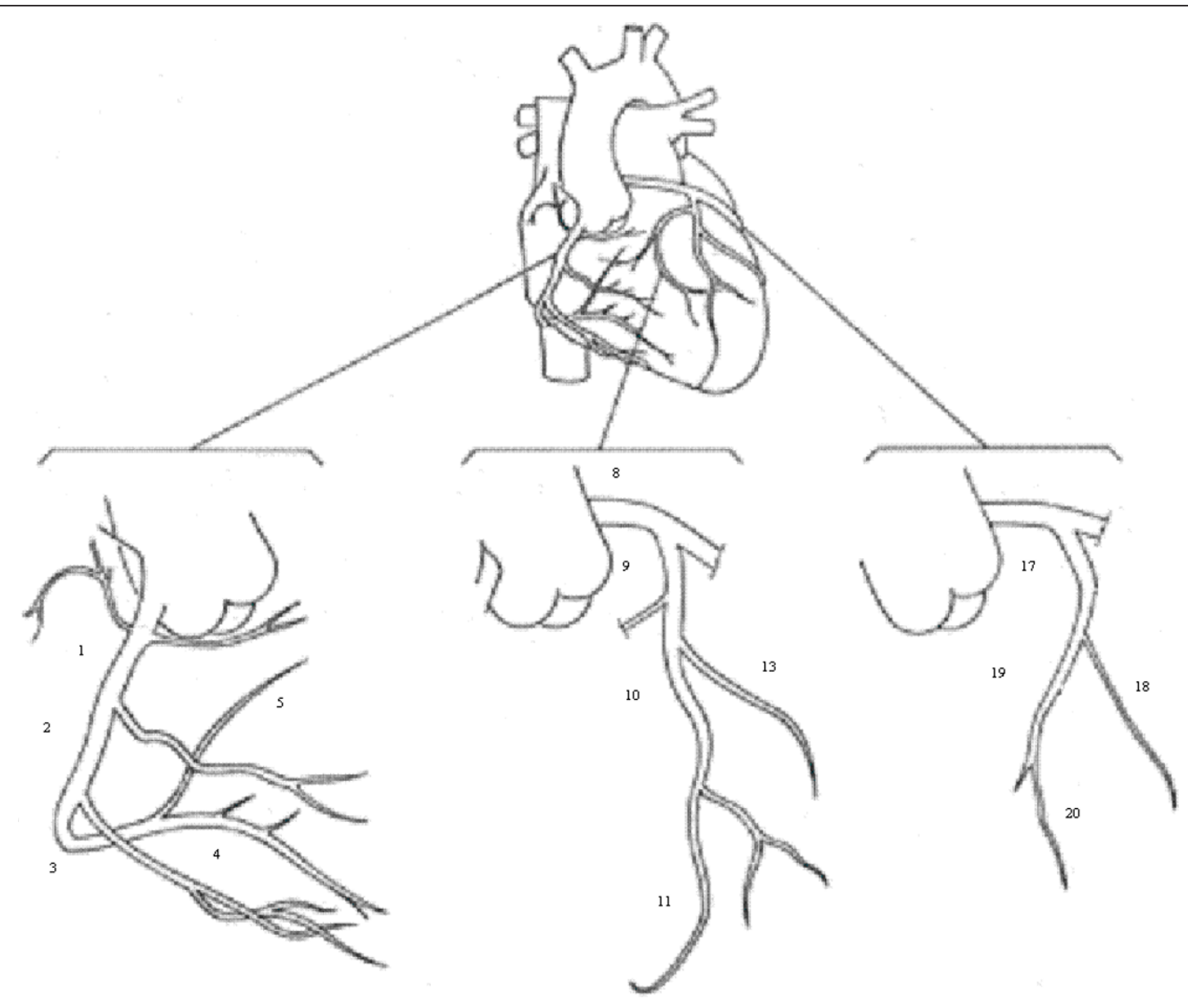

Figure $\mathbf{2}$ Coronary artery map. The figure shows the numerical labeling of segments of the coronary tree.

The displacement type was present in $23 \%$ of segments in the STEMI patients and in $20 \%$ of segments in control patients. $33 \%$ of segments in both the STEMI and control patients had the compression type of CAM. There was no statistically significant difference between the frequency of CAM types of the STEMI and control patients $(\mathrm{p}=0.97)$.

\section{Table 1 Patient Demographics}

\begin{tabular}{lll}
\hline & $\begin{array}{l}\text { STEMI } \\
\text { patients }\end{array}$ & $\begin{array}{l}\text { Control } \\
\text { patients }\end{array}$ \\
\hline Mean age (+/-stdev) & $61(+/-11)$ & $61(+/-12)$ \\
\hline Men (\%) & 80 & 80 \\
\hline Diabetes (\%) & 25 & 25 \\
\hline Current smoker (\%) & 45 & 45 \\
\hline Hypercholesterolemia (\%) & 75 & 55 \\
\hline $\begin{array}{l}\text { Hypertension (\%) } \\
\text { Family history of coronary artery } \\
\text { disease (\%) }\end{array}$ & 55 & 50 \\
\hline
\end{tabular}

Table 2 Frequency of each pattern and type of CAM

\begin{tabular}{lcccc}
\hline Pattern of CAM & \multicolumn{2}{c}{ STEMI patients } & \multicolumn{2}{c}{ Control patients } \\
\hline Non-compression & Number & $\mathbf{( \% )}$ & Number & (\%) \\
\hline Bend & 161 & 67.1 & 164 & 66.9 \\
\hline Bend & 106 & 44.2 & 114 & 46.5 \\
\hline Multiple bend & 12 & 5.0 & 14 & 5.7 \\
\hline Hinge & 77 & 32.1 & 89 & 36.3 \\
\hline Crease & 8 & 3.3 & 8 & 3.3 \\
\hline Wave flex & 9 & 3.8 & 3 & 1.2 \\
\hline Displacement & 0 & 0 & 0 & 0 \\
\hline Lever & 55 & 22.9 & 50 & 20.4 \\
\hline Lineal displacement & 0 & 0 & 0 & 0 \\
\hline Parallel displacement & 31 & 12.9 & 13 & 5.3 \\
\hline Compression & 24 & 10.0 & 37 & 15.1 \\
\hline Compression & 79 & 32.9 & 81 & 33.1 \\
\hline Ostial compression & 8 & 3.3 & 16 & 6.5 \\
\hline Total & 240 & & 245 & \\
\hline
\end{tabular}


The proportion of the compression type of CAM for individual artery segments for both patient groups is shown in Figure 3. The compression type of CAM was more frequent in the proximal and mid segments of the right (\#1 and 2), the left anterior descending (\#9 and $10)$ and to a lesser extent the left circumflex (\#17 and 19) coronary arteries. The compression type was less frequent in the distal segments of the right (\#3,4, and $5)$, left main (\#8), distal left anterior descending (\#11), diagonal (\#13), and the obtuse marginal branches (\#18 and 20) of the left circumflex coronary arteries. No statistically significant difference was found when the frequency of the compression type of CAM was compared between the ST-segment elevation acute myocardial infarction and control patients for the individual coronary artery segments $(\mathrm{p}=0.59)$.

\section{Discussion}

This study shows that the proportion of the compression type of coronary artery motion for individual artery segments is not statistically significantly different between patients who have subsequent STEMIs and age and sex matched controls.

The main limitations of this study are its small sample size and the potential observer bias in the qualitative assessment of CAM. The technique relies on a visual assessment. Knowledge of the asymmetrical frequency distribution of culprit lesions in patients with STEMIs [6] and the presence of stenosis within a segment may bias the qualitative visual assessor.

Although the exact pattern of CAM varies amongst individual patients, there are consistent themes of motion differences between the different coronary arteries $[7,8]$ and along individual arteries [9]. The coronary segments that had high proportions of the compression type of CAM have previously been shown to include the site of most STEMIs $[6,10]$. The distribution in coronary segments of the compression type of CAM for the 40 patients in this study and the location of the culprit lesion responsible for STEMI in 280 patients in a previously published report [6] are shown in Figure 4. The percent compression type of CAM and the percent of culprit lesions per $\mathrm{cm}$ of artery was highest for the proximal and mid parts of the right coronary artery (segments 1 and 2) and the proximal and mid parts of the left anterior descending coronary artery (segments 9 and 10).

Previously published work has shown that the compression type of CAM is a predictor of the location of stenosis [3] and the culprit segment responsible for STEMI $[5,11]$. This study builds on this work by finding no difference in the proportion of the compression type

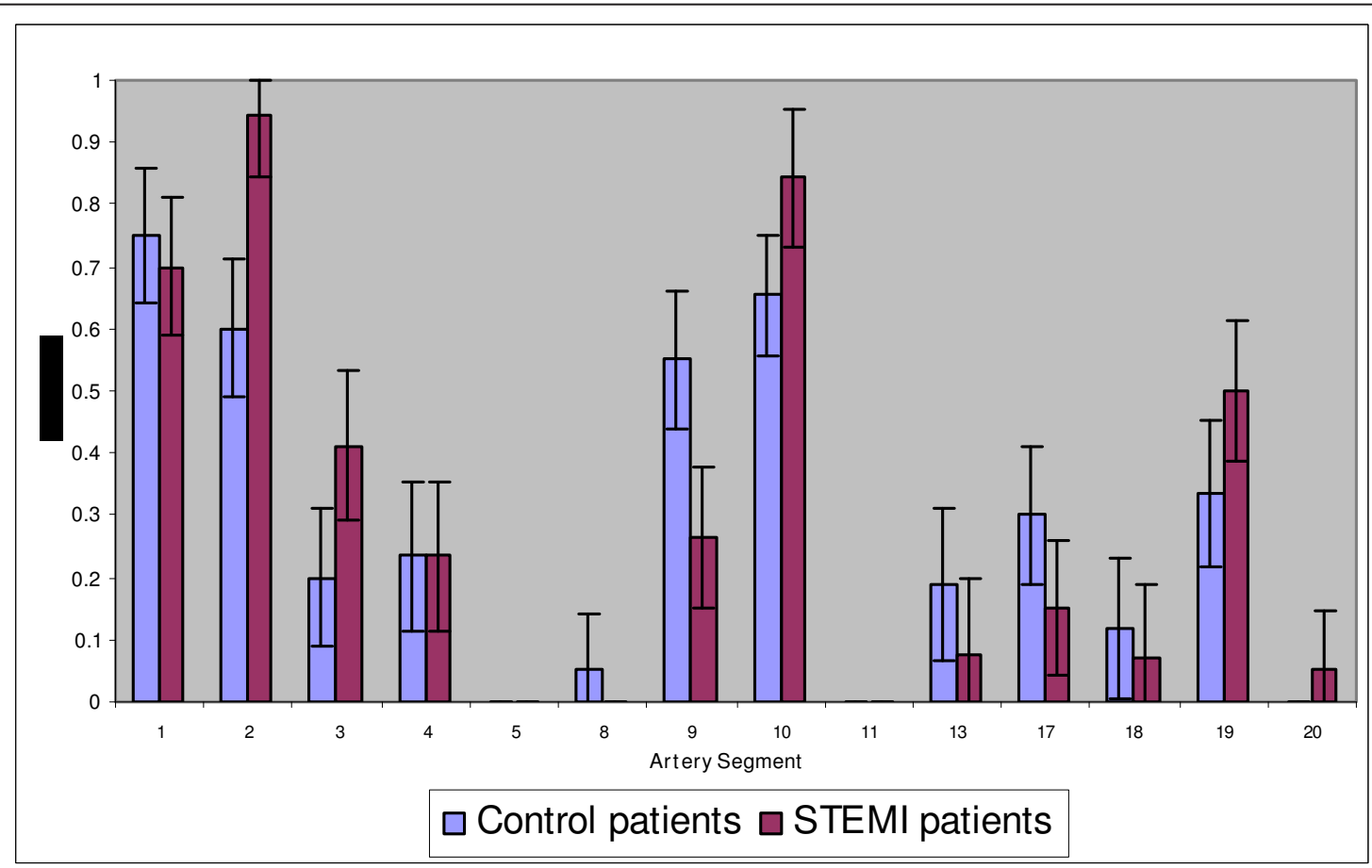

Proportion + - Standard error of the mean.

Figure 3 Proportion of the compression type of CAM for individual artery segments. The segments in the proximal and mid portions of the three coronary arteries had a high proportion of the compression type of CAM in both control and STEMI patients. 


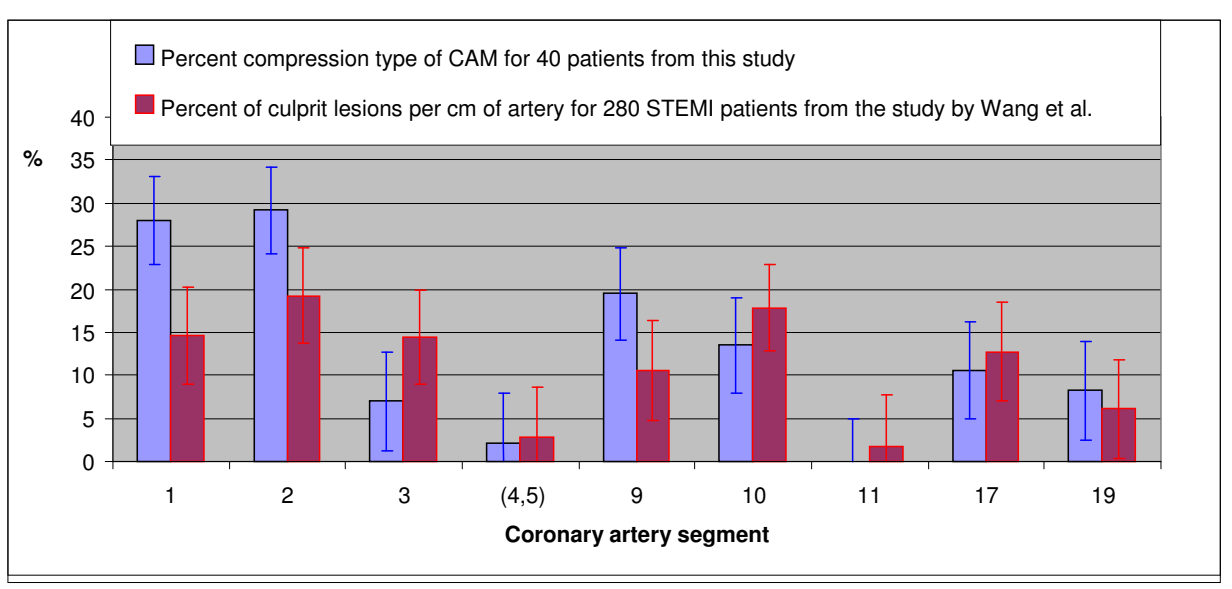

Percent + /- Standard error of the mean.

Figure 4 Comparison of segmental distributions of STEMI and the compression type of CAM. The percentage of culprit lesions per centimeter of artery was calculated from the data for 280 STEMI patients in the study by Wang et al[6]. The percentage of compression type of CAM per centimeter of artery for the 40 patients of this study was calculated by dividing the proportion of the compression type of CAM for the segment by its length in centimeters.

of coronary artery motion between the STEMI population and normal controls. A possible explanation for these findings is that systemic factors determine whether a patient develops coronary atherosclerosis and local biomechanical and/or haemodynamic shear stress determines its location within the coronary arteries.

\section{Acknowledgements}

The authors would like to acknowledge Gai Riddell for retrieval of angiographic data.

\section{Author details}

${ }^{1}$ University of Western Sydney, Sydney, Australia. ${ }^{2}$ Westmead Millenium Institute, Westmead Hospital, Sydney, Australia. ${ }^{3}$ Department of Cardiology, Westmead Hospital, Sydney, Australia. ${ }^{4}$ Department of Cardiology, Liverpool Hospital, Sydney, Australia. ${ }^{5}$ Department of Cardiology, Blacktown Hospital, Sydney, Australia.

\section{Authors' contributions}

$\mathrm{AO}$ and PK conceived the study. $\mathrm{AO}$ undertook the ethics application, data collection, data analysis, and manuscript preparation. KB recommended the statistical methods and supervised data analysis. JF, DR, AH and RD prepared the ethics application, performed manuscript revision and supervised the activities of AO. All authors except KB read and approved the final manuscript.

\section{Competing interests}

The authors declare that they have no competing interests.

Received: 23 September 2010 Accepted: 7 March 2011 Published: 7 March 2011

\section{References}

1. Matter C, Nagel E, Stuber M, Boesiger P, Hess OM: Assessment of systolic and diastolic LV function by MR myocardial tagging. Basic Res Cardiol 1996, 91(Suppl 2):23-28.

2. Rogers WJ, Shapiro EP, Weiss JL, Buchalter MB, Rademakers FE, Weisfeldt ML, Zerhouni EA: Quantification of and correction for left ventricular systolic long-axis shortening by magnetic resonance tissue tagging and slice isolation. Circulation 1991, 84(2):721-731.
3. Konta T, Bett JH: Patterns of coronary artery movement and the development of coronary atherosclerosis. Circ J 2003, 67(10):846-850

4. O'Loughlin AJC, Byth K: The Stretch-Compression Type of Coronary Artery Movement Predicts the Location of Culprit Lesions Responsible for STSegment Elevation Myocardial Infarctions. Heart Lung and Circulation 2007, 16(4):265-268.

5. Chan KH, Chawantanpipat C, Gattorna T, Chantadansuwan T, Kirby A, Madden A, Keech A, Ng MK: The relationship between coronary stenosis severity and compression type coronary artery movement in acute myocardial infarction. Am Heart J 2010, 159(4):584-592.

6. Wang JC, Normand SL, Mauri L, Kuntz RE: Coronary artery spatial distribution of acute myocardial infarction occlusions. Circulation 2004. 110(3):278-284.

7. Gyongyosi M, Yang P, Khorsand A, Glogar D: Longitudinal straightening effect of stents is an additional predictor for major adverse cardiac events. Austrian Wiktor Stent Study Group and European Paragon Stent Investigators. J Am Coll Cardiol 2000, 35(6):1580-1589.

8. Achenbach S, Ropers D, Holle J, Muschiol G, Daniel WG, Moshage W: Inplane coronary arterial motion velocity: measurement with electronbeam CT. Radiology 2000, 216(2):457-463.

9. Ding Z, Friedman MH: Dynamics of human coronary arterial motion and its potential role in coronary atherogenesis. J Biomech Eng 2000, 122(5):488-492.

10. Gibson CM, Kirtane AJ, Murphy SA, Karha J, Cannon CP, Giugliano RP, Roe MT, Harrington RA, Ohman EM, Antman EM: Distance from the coronary ostium to the culprit lesion in acute ST-elevation myocardial infarction and its implications regarding the potential prevention of proximal plaque rupture. J Thromb Thrombolysis 2003, 15(3):189-196.

11. O'Loughlin AJ, Byth K: The stretch-compression type of coronary artery movement predicts the location of culprit lesions responsible for STsegment elevation myocardial infarctions. Heart Lung Circ 2007, 16(4):265-268

doi:10.1186/1756-0500-4-51

Cite this article as: O'Loughlin et al:: The compression type of coronary artery motion in patients with ST-segment elevation acute myocardial infarction and normal controls: a case-control study. BMC Research Notes 2011 4:51. 